\title{
Wearables and the medical revolution
}

\author{
Jessilyn Dunn ${ }^{1,2,3}$, Ryan Runge $\mathrm{R}^{1,2,3}$ \& Michael Snyder*,1 \\ ${ }^{1}$ Department of Genetics, Stanford University, Stanford, CA 94305, USA \\ ${ }^{2}$ Department of Bioengineering, Stanford University, Stanford, CA 94305, USA \\ ${ }^{3}$ Mobilize Center, Stanford University, Stanford, CA 94305 USA \\ *Author for correspondence: mpsnyder@stanford.edu
}

\begin{abstract}
Wearable sensors are already impacting healthcare and medicine by enabling health monitoring outside of the clinic and prediction of health events. This paper reviews current and prospective wearable technologies and their progress toward clinical application. We describe technologies underlying common, commercially available wearable sensors and early-stage devices and outline research, when available, to support the use of these devices in healthcare. We cover applications in the following health areas: metabolic, cardiovascular and gastrointestinal monitoring; sleep, neurology, movement disorders and mental health; maternal, pre- and neo-natal care; and pulmonary health and environmental exposures. Finally, we discuss challenges associated with the adoption of wearable sensors in the current healthcare ecosystem and discuss areas for future research and development.
\end{abstract}

First draft submitted: 14 May 2018; Accepted for publication: 27 June 2018; Published online: 27 September 2018

Keywords: digital health $\bullet$ longitudinal monitoring $\bullet$ sensors $\bullet$ wearables $\bullet$ wireless telemetry

Wearable devices (wearables) are already revolutionizing biomedicine through mobile and digital health by enabling continuous, longitudinal health monitoring outside of the clinic. Previously geared toward consumer health enthusiasts, healthcare practitioners are now beginning to adopt wearables for patient monitoring. Wearables also facilitate algorithm development for automated health event prediction, prevention and intervention. New wearables-based analytic platforms are coming online at an unprecedented rate and are transforming quality and accessibility of healthcare everywhere from hospital intensive care units (ICUs) to in-home chronic disease management to resource-limited field settings. Improved ICU alarms, continuous arrhythmia detection outside of the clinic and rural neo-natal temperature monitoring are just a few of the many recent advancements made possible by wearables. Here, we discuss major innovations in wearable sensing for healthcare, touch upon limitations of existing work and explore future directions (Figure 1A).

Typical wearable devices with applications in health

Many nonimplantable wearable sensors exist for use in consumer health and medical research and some devices are already being integrated into standard clinical practice (Figure 1C). Some common devices are summarized in Table 1. These sensors can generally be classified into three main categories: mechanical, physiological and biochemical (Figure 1B) [1]. The sensor grades range from consumer to clinical to research-grade devices (Figure 1B). Data from these sensors have been used in a wide range of tasks, including, for example, tracking gait, detecting atrial fibrillation and monitoring blood glucose, respectively.

Wearable mechanical sensors usually utilize parts of inertial measurement units (IMUs) to estimate a subject's translational and rotational motion, applied forces and surrounding magnetic field. IMUs use biaxial or triaxial accelerometers to measure planar or 3D movement, respectively, gyroscopes to measure rotation and magnetometers to measure relative position. Accelerometers are most common in wearables, but gyroscopes are appropriate when it is necessary to differentiate acceleration due to gravity versus acceleration due to movement [39]. To obtain exact positioning in space during movement, global positioning systems and altimeters can be used to continually correct for drift errors. Wearable pressure sensors have also become increasingly common to track forces either exerted on or by the body.

Future Medicine 
(A)

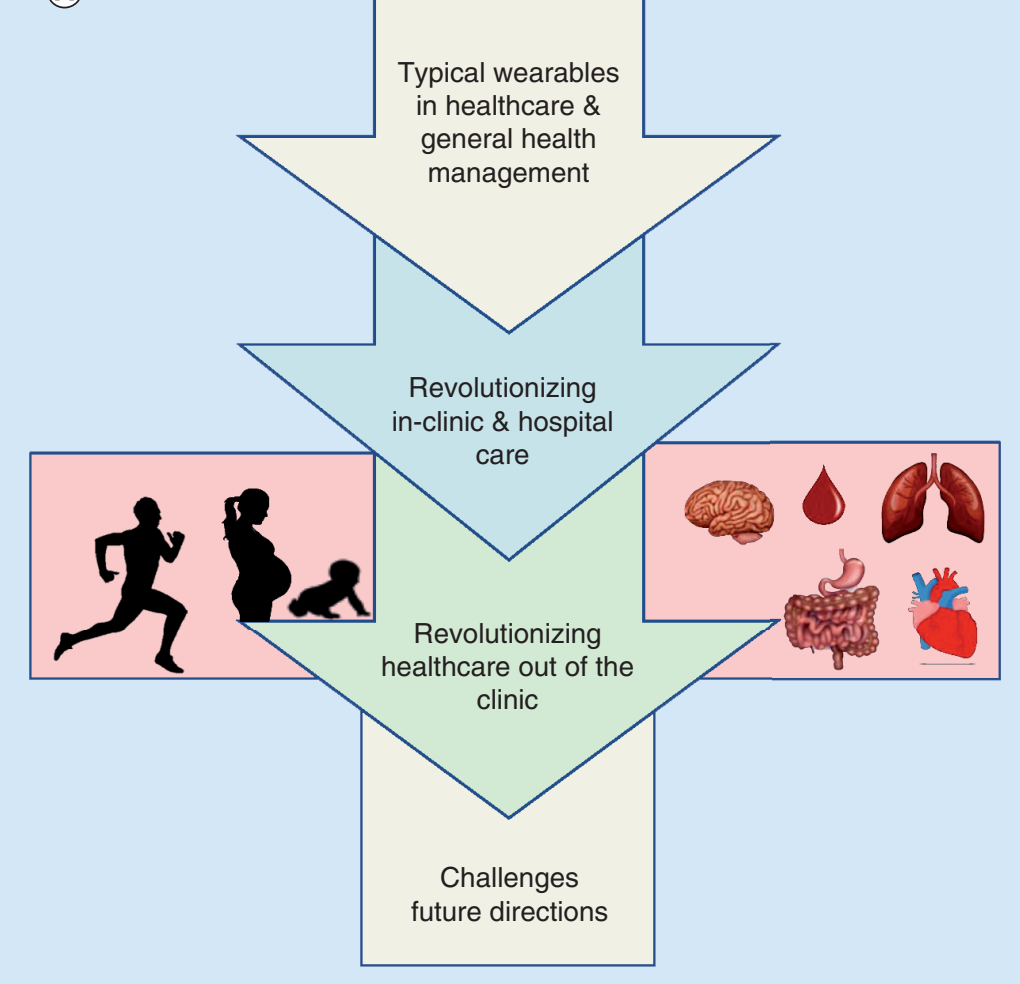

(C)

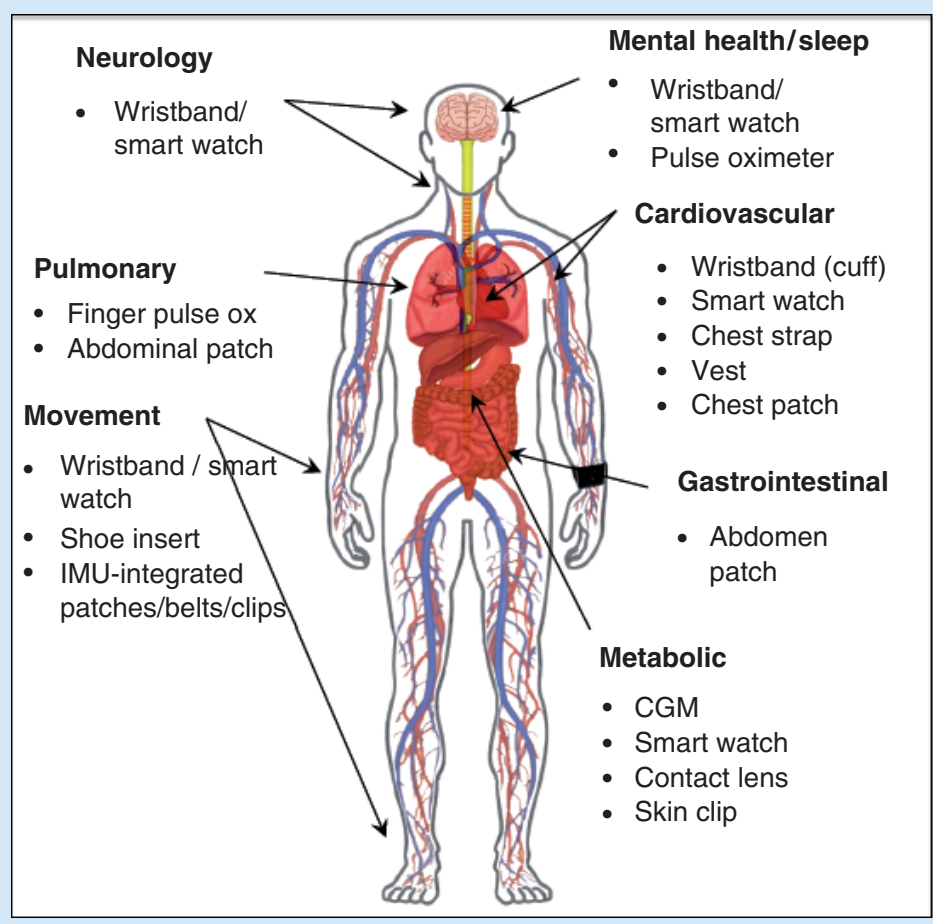

(B)

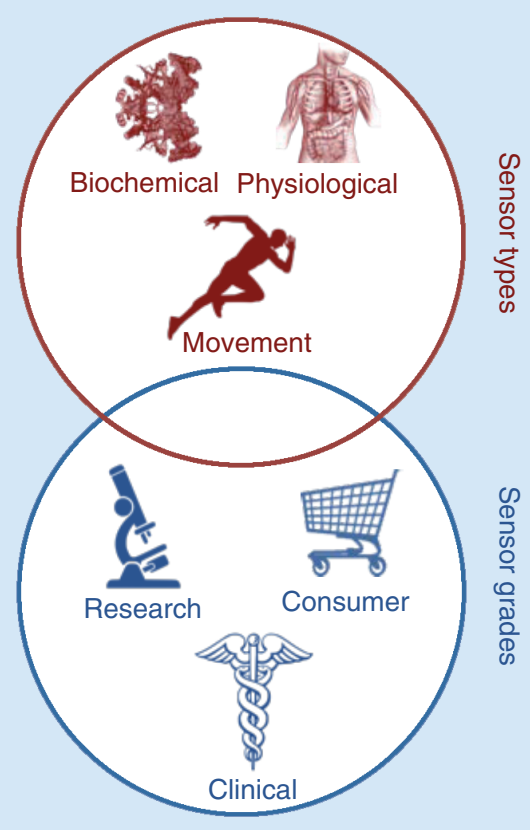

Figure 1. A wide variety of wearable devices facilitate personalized healthcare. (A) Overview diagram of review paper showing major topics covered. (B) The three major wearable sensor types (red) and sensor grades (blue). (C) Wearable devices for use in metabolic, cardiovascular and gastrointestinal monitoring; sleep, neurology, movement disorders and mental health; maternal, pre- and neo-natal care; and pulmonary health and environmental exposures. IMU: Inertial measurement unit; CGM: Continuous glucose monitoring. 


\begin{tabular}{|c|c|c|c|c|c|c|c|}
\hline Manufacturer & Model & Market & Cost (USD) & Form factor & Sensors & $\begin{array}{l}\text { US FDA } \\
\text { status }\end{array}$ & Ref. \\
\hline Abbott & Libre & $\begin{array}{l}\text { Ambulatory diabetes } \\
\text { monitoring }\end{array}$ & $\begin{array}{l}149.98 \text { (cost for } \\
\text { reader and 10-day } \\
\text { sensor) }\end{array}$ & Semi-invasive & CGM & Approved & [2-4] \\
\hline AliveCor & Kardia Band & Consumer & 199 & Wristband & ECG & Cleared & $\begin{array}{r}{[3-} \\
14,15]\end{array}$ \\
\hline Apple & Watch Series 3 & Consumer & 329 & Watch & $\begin{array}{l}\text { Accel, ambient light } \\
\text { sensor, BALT, Gyro, } \\
\text { PPG HR, GPS }\end{array}$ & Precertified & [3-16] \\
\hline Ava Science, Inc. & Ava Wristband & Consumer & 249 & Wristband & $\begin{array}{l}\text { Accel, EDA, PPG HR, } \\
\text { temperature sensors }\end{array}$ & Approved & [17] \\
\hline BACtrack & Skyn & Pre-Market & - & Wristband or watch & Transdermal & - & \\
\hline Bloomlife & $\begin{array}{l}\text { Smart Pregnancy } \\
\text { Tracker }\end{array}$ & Consumer (rental) & 20/week & Abdominal patch & Accel, 3-channel AFE & - & {$[17,18]$} \\
\hline Dexcom & G5 Mobile & $\begin{array}{l}\text { Ambulatory diabetes } \\
\text { monitoring }\end{array}$ & $\begin{array}{l}1016 \text { (cost for } \\
\text { transmitter, receiver, } \\
\text { 4-pack of replaceable } \\
\text { sensors) }\end{array}$ & Semi-invasive & CGM & Approved & {$[2,19]$} \\
\hline Empatica & Embrace & Consumer & 249 & Watch & $\begin{array}{l}\text { Accel, EDA, Gyro, } \\
\text { peripheral } \\
\text { temperature sensor }\end{array}$ & Approved & {$[20,21]$} \\
\hline Fitbit & Charge & Consumer & 149.95 & Watch & Accel, PPG HR & Precertified & [22] \\
\hline Gl Logic & AbStats & Premarket & - & Abdominal device & Vibration, acoustic & - & [23] \\
\hline G-Tech Medical & G-Tech Medical & Premarket & - & Abdominal patch & EMG & - & {$[24,25]$} \\
\hline Health Care Originals & ADAMM-RSM & Premarket & - & Chest patch & $\begin{array}{l}\text { Acoustic, } \mathrm{HR}, \\
\text { temperature }\end{array}$ & - & {$[26-28]$} \\
\hline iRhythm & Ziopatch & $\begin{array}{l}\text { Ambulatory cardiac } \\
\text { monitoring }\end{array}$ & $\begin{array}{l}\text { Ordered through } \\
\text { physician, billed } \\
\text { directly to insurance }\end{array}$ & Chest patch & ECG & Cleared & {$[29,30]$} \\
\hline Med/Wise & Gluco Wise & Premarket & - & $\begin{array}{l}\text { Clip (thumb, } \\
\text { forefinger or } \\
\text { earlobe) }\end{array}$ & $\begin{array}{l}\text { CGM Radio wave } \\
\text { sensor }\end{array}$ & - & \\
\hline Medtronic & Enlite & $\begin{array}{l}\text { Ambulatory diabetes } \\
\text { monitoring }\end{array}$ & - & Semi-invasive & CGM & Approved & {$[5,31]$} \\
\hline Motiv & Motiv Ring & Consumer & 199 & Ring & Accel, PPG HR & - & \\
\hline Omron & Heart Guide & Premarket & - & Watch & $\begin{array}{l}\text { Accel, PPG HR, } \\
\text { oscillometric blood } \\
\text { pressure }\end{array}$ & - & [32] \\
\hline Orpyx & Surro Gait Rx & $\begin{array}{l}\text { Ambulatory gait } \\
\text { monitoring }\end{array}$ & $\begin{array}{l}\text { Ordered through } \\
\text { physician }\end{array}$ & $\begin{array}{l}\text { Watch, shoe insert, } \\
\text { shoe pod }\end{array}$ & Pressure & - & {$[33$} \\
\hline JOrpyx & Surro Sense Rx & $\begin{array}{l}\text { Ambulatory gait } \\
\text { monitoring }\end{array}$ & $\begin{array}{l}\text { Ordered through } \\
\text { physician }\end{array}$ & $\begin{array}{l}\text { Watch, shoe insert, } \\
\text { shoe pod }\end{array}$ & Pressure & Cleared & [34] \\
\hline Oura & Oura Ring & Consumer & 299-999 & Ring & $\begin{array}{l}\text { Accel, Gyro, PPG HR, } \\
\text { skin temperature }\end{array}$ & - & [35] \\
\hline Preventice & Bodyguardian Heart & $\begin{array}{l}\text { Ambulatory cardiac } \\
\text { monitoring }\end{array}$ & $\begin{array}{l}\text { Ordered through } \\
\text { physician billed } \\
\text { directly to insurance }\end{array}$ & Chest patch & Accel, ECG & Cleared & [36] \\
\hline Sentio Solutions & Feel & Premarket & 149 & Wristband & $\begin{array}{l}\text { EDA, PPG HR, skin } \\
\text { temperature }\end{array}$ & - & [37] \\
\hline Tempdrop & Tempdrop & Consumer & 150 & Underarm armband & Thermometer & - & \\
\hline Verily & Alcon & Premarket & - & Smart lens & CGM & - & \\
\hline VitalConnect & Vital Patch & Premarket & - & Chest patch & $\begin{array}{l}\text { Accel, ECG, } \\
\text { thermistor }\end{array}$ & Cleared & [11] \\
\hline Yono & Earbud & Consumer & 149.99 & Earbud & Thermometer & - & \\
\hline Zoll & Lifevest & $\begin{array}{l}\text { Ambulatory cardiac } \\
\text { monitoring/interventio } \\
\text { (rental) }\end{array}$ & $\begin{array}{l}3-4 k \\
\text { on }\end{array}$ & Vest & ECG & Approved & [38] \\
\hline
\end{tabular}

Accel: Accelerometer; AFE: Analog front end; BALT: Barometric altimeter; CGM: Continuous glucose monitor; ECG: Electrocardiography: EDA: Electrodermal Activity; EMG: Electromyography; GPS: Global positioning system; Gyro: Gyroscope; HR: Heart rate; PPG: Photoplethysmography; Therm: Thermometer. 


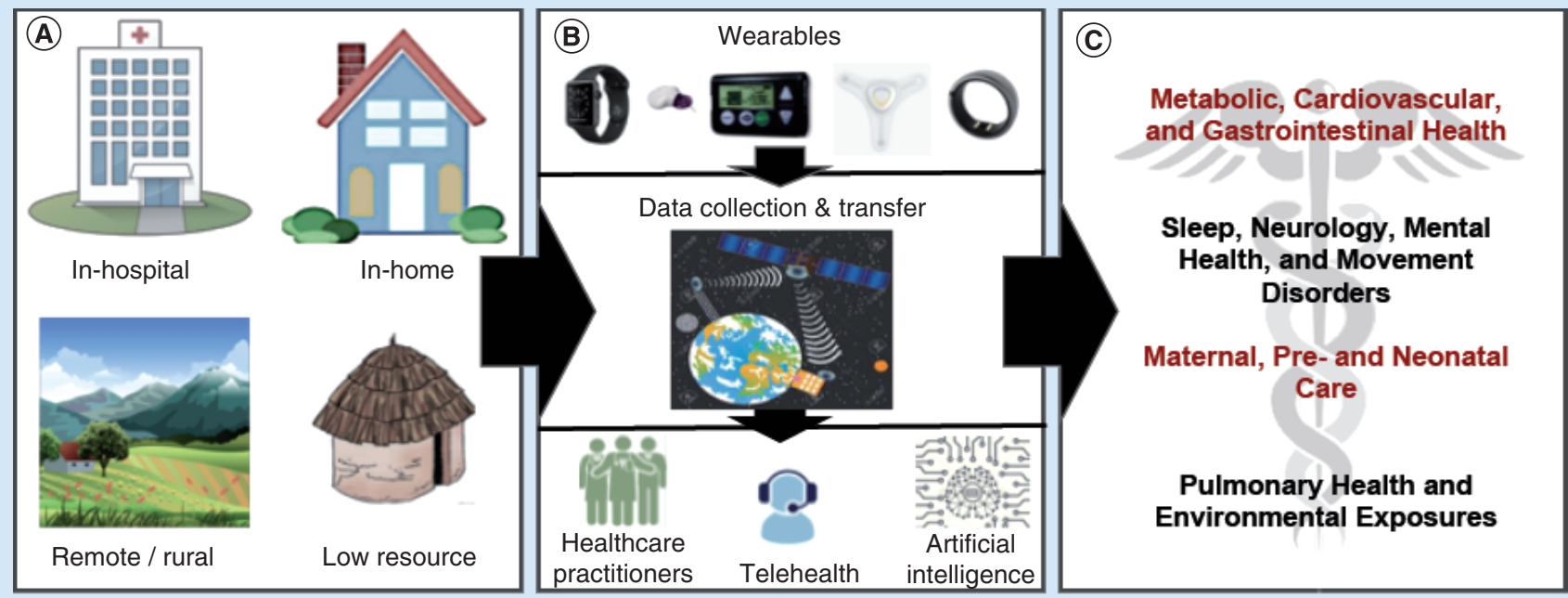

\begin{tabular}{|ll|}
\hline (D) Challenges \& Limitations & - Accessibility \\
- Accuracy & - Cost \\
- Privacy \& security & - Compatibility \\
- Oversight & - Acceptability \\
- Scientific peer-reviewed & - Interpretation \\
& evidence for safety \& efficacy in \\
healthcare & - Technological/form factor \\
& - Lack of standards \\
\hline
\end{tabular}

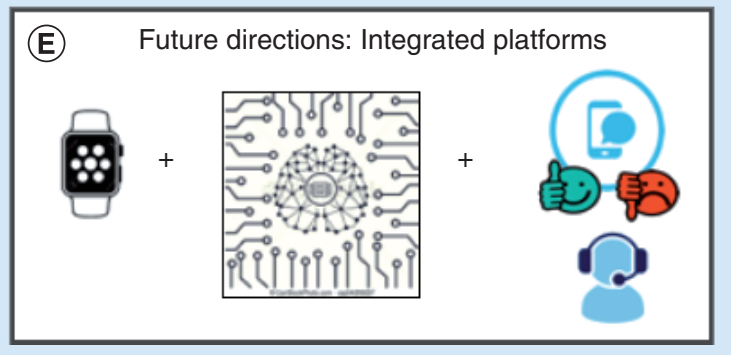

Figure 2. The wearables landscape may impact their adoption in clinical care. (A) Settings where wearables can provide or improve healthcare. (B) Flow of data from wearables to health decision-makers. (C) Areas of healthcare that currently existing wearables can target (some with strong evidence supporting their use and others are newer). (D) Challenges and limitations for the adoption of wearable technology in healthcare. (E) A single platform integrating wearable device data collection, analytics and intervention delivery will constitute a complete operating healthcare monitoring system.

Although mechanical sensors usually use piezoelectric components to convert mechanical motion into electrical signals, physiological sensors use optical, electrical, acoustic or thermal sensing components to measure biological signals including vital signs (e.g., heart rate, temperature, BP, blood oxygen saturation), bodily functions (gut and respiratory activity) and bioelectrical activity (e.g., bioimpedance and electrodermal activity, ECG, electromyography, electroencephalography). Photoplethysmography (PPG) is a common sensor used to detect heart rate via changes in light absorbance through thin tissues. Bioimpedance sensors are thought to be useful for detecting stress and emotion through changes in electrical resistance of tissues related to neural activity.

Wearables that are most commonly related to clinical applications include biochemical sensors. Biochemical sensors have a wide range of uses including glucose, alcohol, electrolyte, $\mathrm{pH}$, oxygenation/gas and humidity sensing. Biochemical sensors combine a chemically sensitive layer and transducer to convert a chemical or biological analyte into an electrical signal. These sensors are used to measure both internal and external chemical and biochemical species for health monitoring.

Wearable devices used for general health management

Wearable devices provide opportunities to improve healthcare in a variety of settings, from in-hospital and in-clinic care, to ambulatory care in the home and in remote geographical settings including rural areas and low-resource environments (Figure 2A). One of the major challenges in the wide adoption of wearable biosensor technologies for healthcare is the extraction of useful and actionable health-related information from the large volume of data. To address this data deluge, we and others have recently developed algorithms to automatically process and interpret wearables data in order to present evidence-based health insights such as detection of inflammation or prediction of cardiometabolic health based on activity habits [40-43]. This emerging work is anticipated to grow in scope and 


\begin{tabular}{|c|c|c|c|c|}
\hline & Lyme disease & Respiratory viral infection & Insulin resistance & Atrial fibrillation \\
\hline \multirow[t]{3}{*}{ Wearable device sensors } & Resting HR (PPG) & Resting HR (PPG) & $\begin{array}{l}\text { Diumal HR difference } \\
\text { (PPG) }\end{array}$ & HR (AliveCor ECG) \\
\hline & $\begin{array}{l}\text { Skin temperature } \\
\text { (Thermopile) }\end{array}$ & $\begin{array}{l}\text { Skin temperature } \\
\text { (Thermopile) }\end{array}$ & $\begin{array}{l}\text { Physical activity } \\
\text { (accelerometer) }\end{array}$ & $\mathrm{HR}(\mathrm{PPG})$ \\
\hline & $\mathrm{SpO}_{2}$ (Pulse Oximeter) & & & \\
\hline ML algorithm & $\begin{array}{l}\text { Peak detection; logistic } \\
\text { regression }\end{array}$ & $\begin{array}{l}\text { Sliding window peak } \\
\text { detection; logistic } \\
\text { regression }\end{array}$ & Multiple regression & Deep neural network \\
\hline
\end{tabular}

dramatically revolutionize the healthcare landscape around the globe by providing opportunities for automated detection of health events and deployment of mobile interventions (Figure 2E).

Mobile health technologies also offer an opportunity to scale physiological monitoring studies to uncover new uses for wearables in healthcare. Data analysis methods, including machine learning, show promise to expand the use of wearables into clinical applications that range from detection of acute health events (e.g., infection/inflammation) to monitoring and management of chronic diseases (e.g., cardiovascular disease and diabetes) (Table 2) [40,41]. In our study on the utility of wearable sensors for managing health and diagnosing disease, participants wore devices including consumer fitness trackers. These devices measured physical activity, heart rate, skin and ambient temperature, electrodermal activity, blood oxygen saturation and radiation exposure [41]. We concomitantly performed comprehensive clinical and biomolecular measurements including multi-omics as a part of the Integrative Personal Omics Profiling study [40,44-46]. Encouragingly, using only measurements from a consumer smart watch, we were able to predict clinical measurements of inflammation, infection and insulin sensitivity status based only on measurements from a consumer smart watch [40]. Others have also demonstrated the utility of consumer fitness trackers for health-related insights and personalized interventions. Examples include the correspondence of wearables-derived resting heart rate and activity with cardiovascular and metabolic disease markers, and wearables data utility in designing behavioral coaching $[40,42,43]$. Building upon the idea of multimodal wearable sensor data integration, industry players have begun developing platforms in this space. PhysIQ is one example of a platform that detects subtle changes from a patient's baseline using biosensing devices to provide real-time health insights [47].

Clinical biomarkers are measurable indicators of biological state that can aid in disease diagnosis, prognosis and treatment effects. Wearables can also generate such indicators. Digital biomarkers are digitally collected data, such as heart rate from a wearable device, that are transformed through fmathematical models into indicators of health outcomes like prediabetes (Table 2). Some digital biomarkers have been found to outperform traditional clinical methods, for example, for arrhythmia detection, because of their ability to continuously monitor patients outside of the clinic. The most successful digital biomarkers have been developed based on supervised, unsupervised and semi-supervised machine learning models. One major obstacle of using supervised machine learning for digital biomarker discovery is the amount of costly labeled data required to train a supervised model [41]. A semi-supervised method that reduces the amount of labeled wearables data needed for training digital biomarker models by tenfold was developed to address this challenge. This method incorporates pretraining a long short-term memory sequence autoencoder with unlabeled wearables data [41]. Cardiogram's DeepHeart platform also takes advantage of a semisupervised approach. A partnership between Cardiogram and the University of California San Francisco Health eHeart study explored detecting clinical conditions from wearables data in 14,011 wearable-equipped participants. This team has demonstrated early success in detecting Type II diabetes (85\% accuracy), atrial fibrillation ( $97 \%$ accuracy), sleep apnea (90\% accuracy), hypertension ( $82 \%$ accuracy) and more recently, cardiovascular risk using the DeepHeart platform [42,48]. VitalConnect (CA, USA) has also built successful sleep apnea and fall detection algorithms using respiratory rate and accelerometry [48-51].

\section{Wearable biosensors revolutionizing in-clinic/hospital care}

Wearables with continuous data collection, real-time analytics and presentation of actionable health insights provide unprecedented opportunities for revolutionizing care in clinical and hospital settings. Historical device data collected prior to patient presentation in a clinic or emergency department $(\mathrm{ED})$ facilitate rapid triaging to provide immediate 
care to those patients most in need. Researchers have received favorable feedback on the perceived usefulness of wearable monitoring in emergency room settings from patients and nurses [52].

Recent studies explored the utility of vital signs for automated triage of ED patients. Levin et al. built a machinelearning-based system called e-triage that uses incoming patient vital signs, chief complaint and active medical history to automate $\mathrm{ED}$ triage. In a multisite retrospective study of $172,726 \mathrm{ED}$ visits, e-triage predictions had areas under the receiver operating characteristic curve ranging from 0.73 to 0.92 . E-triage is currently implemented at Johns Hopkins Medicine for prospective validation studies [53]. Moreno et al. extended this concept by developing wearable bracelets for patients admitted to the ED, although these devices have not yet been tested for efficacy of patient triage [53,54].

For hospital inpatient care, continuous monitoring has been used for decades through multiparameter patient vital signs monitors (e.g., BP, blood oxygen saturation [ $\left.\mathrm{SpO}_{2}\right]$, heart rate, respiration rate) [55]. However, continuous monitoring of nonbedridden patients is more challenging because patients must travel around the hospital with bulky monitoring devices [22]. The wireless nature of new wearable sensors that measure the aforementioned vital signs as well as additional parameters (e.g., movement, skin conductance, food intake, self-care and bathroom habits, etc.) provide an unprecedented opportunity to dramatically improve patient monitoring in clinical and hospital settings while also improving patient comfort and mobility [22].

ICUs are already benefiting from algorithms that synthesize multimodal sensor data and tailor alarm systems and medical decision support systems. By continuously monitoring for dynamic changes to patient status, warning signs prior to health event escalation may provide the opportunity for early interventions to prevent severe health events [22]. Another critical area this can addresses is alarm fatigue in the ICU. In a recent feasibility study, the Fitbit Charge (CA, USA) was used to monitor post-ICU convalescence in 50 stable ICU patients over $24 \mathrm{~h}$. Heart rate, arrhythmia detection and sleep measurements from the Fitbit were compared against clinical gold standards. Tachycardia detection had $69.5 \%$ sensitivity and $98.8 \%$ specificity [22]. To improve the positive predictive value of ICU alarms, Aboukhalil et al. developed a novel method for suppressing false arrhythmia alarms in the ICU using continuous ECG and arterial BP waveforms [56]. CDSs are software aids for clinical decision making (e.g., Medtronic VitalSync, MN, USA) [57,58]. Integration of wearables data in CDS can improve their utility and outcomes. Challenges of integrating wearables into CDS include utilizing training data that is appropriate for the patient population, testing implementation of the CDS for specific clinical outcomes and summarizing and retuning results to clinicians and patients [59-62]. Wearables can also revolutionize telemedicine by providing information to remote physicians that enhances the clinical snapshot collected while the patient is in the hospital.

To implement wearables in the clinic, methods must be developed to integrate wearables data in a meaningful way into the electronic health record. Recent efforts include partnerships announced since 2014 between companies that aggregate wearable device data with electronic health record/interoperability companies. These partnerships include Apple Healthkit and Epic, Validic and Cerner, the MyCarolinas Tracker App and Canopy, AliveCor (CA, USA) and Practice Fusion, Medable and Redox, Healow and eClinicWorks, and NantHealth, American Well and Allscripts. At this time, most integration/interoperability apps aggregate data from hundreds of different wearables with their partnered electronic health record systems. With this integration comes advances in defining and transporting protected health information from wearables and smart phone apps to electronic health records, and improvements in clinical trial systems. For example, Precision Digital Health has developed the SUMMA platform, which uses standards from the Health Level 7 Fast Healthcare Interoperability Resource to integrate wearables with electronic health records to improve clinical trial design and outcomes. Electronic health record integration raises challenges surrounding interpretation of wearables data by clinicians, development of real-time interventions, maintenance of data privacy and security, approval by the US FDA and on-off-label use of the data, and initiation and maintenance of patient participation.

\section{Wearable biosensors revolutionizing specific fields of healthcare outside of the hospital \& clinic}

Outside of the clinic, wearable sensors have found utility in a variety of healthcare-related applications, including general wellbeing, broad medical care, condition-specific monitoring and mobile health interventions. In the following section, we discuss wearables used outside of the clinic for metabolic, cardiovascular and gastrointestinal monitoring; neurology and mental health; maternal, pre- and neo-natal care; and pulmonary health and environmental exposures (Figures 1A \& C \& 2C). 
Metabolic, cardiovascular \& gastrointestinal health

Metabolic syndrome is marked by a cluster of conditions including high BP and blood sugar, abdominal obesity and abnormal cholesterol or triglyceride levels that often occur together and increase the risk of cardiovascular disease, stroke and diabetes [31].

As diabetes and metabolic syndrome increase in prevalence, methods to continuously monitor blood glucose have improved dramatically. The first continuous glucose monitor (CGM) for retrospective patient analysis (MiniMed) earned FDA approval in 1999. The leading CGMs on market today are Dexcom (CA, USA), Medtronic (MN, USA) and Abbott (IL, USA) devices [2-5,19,31]. All are wearable but invasive to some extent (through a subdermal needle or flexible filament insertion). Still on the horizon are noninvasive CGMs which provide promise for individuals with prediabetes or noninsulin-dependent Type II diabetes for chronic disease management and prevention, as well as for Type I diabetics for integration with insulin pumps to create an 'artificial pancreas'. Noninvasive CGMs undergoing research on longer horizons include smart watches and contact lenses [6-8]. Two established partnerships pursuing the tear-based glucose sensing market include Verily/Alcon (CA, USA) and Noviosense/Gentag/Mayo Clinic/Fraunhofer Institute for Microelectronic Circuits and Systems. GlucoWise (London, UK) is another device under development promising noninvasive CGM by transmitting high frequency, low-power radio waves through a thin section of skin with high blood flow (e.g. earlobe or area between the thumb and forefinger).

Wearables have been highly successful for exploring cardiac health and detecting arrhythmias outside of the clinic. Improvements upon Holter monitoring, the standard of care for ambulatory monitoring for cardiac events outside of the clinic since 1965, include replacing lead-based monitoring to improve adherence and data collection and extending the time period of continuous monitoring with alternative form factors, although the data types and accuracy may be different [9]. The most successful replacement technologies to date include three form factors: temporary passive monitoring (usually 1-2 weeks) using a patch (e.g., iRhythm's ZioPatch, Preventice's Bodyguardian Heart, VitalConnect's VitalPatch), continuous but impassive monitoring (e.g., AliveCor's KardiaMobile or KardiaBand or continuous passive monitoring using optical heart rate sensors (e.g., smart watch PPG sensor combined with an analytics engine like cardiogram, which has atrial fibrillation detection of 97\% accuracy) [10-14,29,30,63-65]. AliveCor's KardiaBand is a ECG-capable smart watch band that can be paired with smartwatch PPG for continuous, passive atrial fibrillation monitoring [15]. Kardias 30-s ECG is initiated by the user and is not continuously or passively collected. A recent trial comparing AliveCor's technology with routine care $(\mathrm{n}=1001)$ resulted in a nearly fourfold increase in atrial fibrillation diagnosis [15]. Companies like Zoll (MA, USA) have combined arrhythmia detection technology with an interventional wearable defibrillator, called LifeVest, to automatically revive patients in the case of a cardiac event [38]. LiveVest showed comparable outcomes to implantable cardiac devices in a 3-year study $(\mathrm{n}=3569)$ with acute event survival at $99.2 \%$ [38]. To improve cardiac rehabilitation, a smart phone system was developed to recommend exercise intensity by comparing real-time wearable heart rate data with a predefined target heart rate zone, enabling patients and their care providers to monitor recovery and general cardiovascular health over time [66].

Hypertension affects at least one in three US adults and is a growing problem around the world [67]. Diagnosis and monitoring of hypertension and treatment efficacy is difficult for a few reasons: a single blood pressure (BP) measurement in the clinic often does not capture true average BP, BP fluctuates throughout the day based on circadian rhythms and daily activity, and the 'white coat syndrome' can lead to higher BP measurements in the clinic [68-73]. In order to measure BP continuously over the course of the day and longitudinally over time, work is being done to replace current technologies that require cuff-based oscillometric measurements [74]. Small and affordable BP monitors like table-top (e.g., Omron) and cuff-based wearable ambulatory BP monitors (e.g., Qardio Arm) enable in-home monitoring, but are neither continuous nor passive. New devices that promise to change the current paradigm of hypertension monitoring use either inflating wristband cuffs (e.g., Omron HeartGuide, currently undergoing clinical testing) or optical pulse wave-based metrics of BP [75,76]. If FDA approved, these devices will pioneer the field of continuous BP monitoring. Other BP monitoring methods used non-FDA-approved oscillometric methods or case prediction algorithms based on heart rate and steps [32].

Gastrointestinal diseases often co-occur with metabolic syndrome, but the two conditions may also occur independently [77,78]. Digestive disorders and gastrointestinal diseases affect the GI tract, liver, pancreas and gallbladder and can affect individuals in an acute or chronic manner. The total direct and indirect cost of care for gastrointestinal disorders is estimated at $\$ 142$ billion a year [79]. Wearable technologies to detect, diagnose and manage gastrointestinal conditions are limited. The Gastrointestinal Logic AbStats system (CA, USA) provides real-time 
digestive telemetry via noninvasive vibration and sound sensing to assess postsurgery GI tract re-engagement [23]. G-tech (Mountain View, CA, USA) takes a different approach to gastric myoelectric activity monitoring via electrodes that measure motor activity of the stomach, small intestine and colon [24,25]. Researchers at the University of California San Diego have significantly expanded the potential for gastric monitoring via electrogastrogram [80-82]. An early-stage ingestible sensor is being explored to diagnose gastrointestinal slowdown or monitor food intake via gastrointestinal movement [83]. This device adheres to the stomach wall or intestinal lining, where it can measure the rhythmic contractions of the digestive tract. The devices described above provide new sensing and potential therapeutic modalities and are expected to dramatically improve gastrointestinal care.

\section{Sleep, neurology, mental health \& movement disorders}

Early diagnoses and improved patient monitoring for sleep, neurology and mental health are becoming possible with continuous monitoring afforded by wearable sensing. 70 million Americans experience chronic sleep problems. Polysomnography, the current gold standard for sleep monitoring, often conflicts with subjective self-reports of sleep and is difficult to monitor longitudinally [84]. Wearable sensors show promise for monitoring sleep and related outcomes. The Oura ring (FIN) compared with polysomnography had a 96\% sensitivity to detect sleep, and agreement of 65, 51 and 61\%, in detecting 'light sleep', 'deep sleep' and REM sleep, respectively. Specificity in detecting wake was $48 \%$. Similar to PSG, 'deep sleep' detected with the Oura ring was negatively correlated with advancing age [35]. Obstructive Sleep Apnea (OSA), one of the most common sleep disorders, is estimated to affect up to $7 \%$ of adults in the USA [85,86]. Wearables have been successful in detecting OSA in a variety of ways, including with personalized machine learning algorithms based on similarity to other OSA patients (detection accuracies between 90 and 93.6\%) [41,87]. Sleep impacts chronic illness, neurological conditions and acute cognitive function including memory and mood [22,88-90]. Diminished motor-skill coordination from lack of sleep leads to accidents and injury, and chronic sleep deprivation is linked to chronic illness such as heart disease, obesity, diabetes and depression [85]. To explore the use of wearable devices to detect drowsiness, one recent pilot study $(\mathrm{n}=10)$ used a single-channel electroencephalogram and IMU sensor to detect five different levels of drowsiness with an average of $95.2 \%$ accuracy [91]. In our recent study monitoring physiological changes using wearables during flight, we found that $\mathrm{SpO}_{2}$ directly corresponds to drowsiness level [40].

Mood disorders are top medical causes for disability [92]. Prevalence of depressive disorders increased $30 \%$ from 1990 to 2010 and one in three veterans experience symptoms of depression [92,93]. Mobile apps provide a means to more accurately record symptoms (e.g., depressive mood, anxious mood or fatigue) through real-time assessments that can be administered outside of the clinic and accurate recall and time of recording [94,95]. Still a challenge is the dependence on active patient participation and the semi-quantitative nature of the measurements. Continuous, passive wearables monitoring can lower dependence on participant interaction and increase the volume of data that can be collected over time. Additional relevant data types can also be collected. A study on the relationship between mood and physical activity using wearable actigraphs $(n=85)$ showed that distinct activity patterns correspond with worsening depressive symptoms [96]. Bipolar Disorder (BD) mood states including depression, mixed, mania and euthymia were accurately classified (68-85\%) using a support vector machine trained on features of heart rate variability and previous mood $(\mathrm{n}=8)$ [97]. Wearables may also differentiate between disorders that have shared symptoms which may cause delays in diagnosis or treatment, imprecise treatment or misdiagnosis (e.g., hyperactivity shared by BD and ADHD). By tracking additional metrics like diurnal activity and circadian amplitude and dysregulation using wearable sensors, researchers could distinguish BD and non-BD conditions, such as ADHD, with $83 \%$ accuracy (92\% specificity and $64 \%$ sensitivity) [98].

With the ability to more accurately track acute and chronic neurologic and mental health symptoms, the opportunity for real-time intervention follows. Artificial intelligence platforms can learn and predict effective interventions for an individual using a combination of personal historical data and population-level data to optimize treatment type and timing. One example is Sentio Solutions' Feel wristband (San Francisco, CA, USA), which is anticipated to deliver personalized interventions to the wearer according to their mood state based on their proprietary neural network model that can accurately detect $70-75 \%$ of relaxed, anxious, excited and fun mood states using features of heart rate variability and skin conductance $(n=224)$ [37]. Another example is a platform for emotion self-regulation for individuals with Autism Spectrum Disorder. Using physiological and movement metrics from a smartwatch to infer outburst patterns, real-time, self-regulation exercises are sent as smartwatch notifications as a just-in-time intervention. In a recent study, 33 and $43 \%$ of the alerts successfully engaged the participant in the self-regulation exercise and resulted in de-escalation of a potential episode, respectively [99]. Technological 
limitations include noisy heart rate during periods of high intensity activity. Other nonwearable technologies for Autism Spectrum Disorder interventions include computer vision monitoring [100].

Several neurologic disorders include motor control dysregulation. Epilepsy, which can develop at any stage of life, affects more than 50 million globally [101,102]. The Empatica Embrace (MA, USA), a smartwatch that evolved out of the MIT Media Lab, can detect seizures using electrodermal activity and accelerometry and can also notify emergency contacts $[20,21,103]$. In a study of 80 pediatric patients with varying degrees of epilepsy, Embrace detected 94\% of GTC seizures with less than one false alarm per 24-h period [20]. Orpyx Medical Technologies, Inc. (AB, CAN) designed the SurroGait Rx shoe insole and vest system to measure and correct gait in multiple sclerosis (MS) patients. The system senses and converts pressure produced on the insole during walking to the vest with a built-in vibrotactile array to re-teach multiple sclerosis patients to walk $(\mathrm{n}=7,16$ weeks) [33]. This device has also been successful for pressure-offloading alerts to prevent diabetic foot ulcers, which cost the US $\$ 81.7$ billion in $2012[104,105]$. Gait analysis is also used to assess Parkinson's Disease (PD) patients and generally requires video-based motion-capture in a specialized laboratory [34]. IMUs affixed to shoes were recently found to result in comparable diagnoses with gold standard video-based assessments to distinguish gait differences between PD and unaffected patients and for longitudinal tracking of gait changes in PD $(n=291)$ [78]. Wearable sensors have been highly successful in fall detection $[50,106,107]$. A study on VitalConnect's HealthPatch wearable found that a tri-axial accelerometer worn at any location of a participant's body, and in any orientation, could detect falls with a sensitivity of $99 \%$ and specificity of $100 \%(n=25)[50]$.

Maternal, pre- \& neo-natal care

There are approximately six million pregnancies each year in the USA and pregnancy-related complications are common: one in ten infants in the USA are born preterm and there has been a steady increase in pregnancyrelated complications and mortality from 1987 to 2013 [108-113]. Further, $6.2 \%$ of women and $11.5 \%$ of men of reproductive age have impaired fertility [114,115]. Improved screening methods are under development, and studies show that remote monitoring and telemedicine-based approaches greatly improve care access for women with newborns [116-118]. Here we summarize devices on market for fertility tracking and pre-, neo-natal and maternal monitoring (Figure 1C, right).

To improve odds of conception, fertility tracking devices have been developed specifically for use in women to track ovulation based on physiological changes like body temperature. The Ava wristband (San Francisco, CA, USA) is FDA approved for fertility tracking and measures nine physiological parameters including resting pulse rate, skin temperature, heart rate variability, sleep, breathing rate, movement, perfusion, bioimpedance and heat loss. In a recent study of 91 women, a significant increase of the fertile-window sleeping pulse rate compared with the menstrual phase (an average of 2.1 beat-per-minute) measured via PPG was found [17]. Among wearable temperature trackers, the Yono earbud (CA, USA) and Tempdrop underarm armband (ISR) measure basal body temperature at night to track ovulation cycles. These technologies are also being explored for contraception, for example, paired with algorithms like Natural Cycles [119].

Several devices exist for tracking fetal heart rate and movement, although none had been FDA approved at the time of this review. The Bloomlife Smart Pregnancy Tracker (CA, USA), a wearable device for tracking contractions in the third trimester of pregnancy, was shown to have an $87 \%$ success rate in predicting delivery within $24-\mathrm{h}$ using electrohysterography and HR $[18,120]$. Their labor risk score was developed based on in-hospital data from 55 pregnant women and was evaluated on recordings from 142 pregnant women in free-living conditions. The Moodo, a wearable device, and the Bellabeat Shell, a nonwearable smart phone attachment, record and amplify sounds to track fetal heart rate and count fetal kicks. Neo-natal and infant physiologic monitoring to assist parents and caregivers come in many different form factors to accurately monitor infant respiration, pulse rate and blood oxygen saturation, and to generate alarms for apnea, tachycardia, bradycardia and desaturation [121]. Bempu is a neonatal bracelet used in low-resource settings to monitor premature infant temperature to detect hypothermia [122]. Physiologic monitors that include sensing for pulse rate, respiratory motion and/or pulse oximetry include the Baby Vida and Owlet smart socks, the Mimo onesie, the MonBaby and Snuza Pico button/clip, and the Sproutling leg band. Such devices have caused frustration in the pediatric medical community due to unsubstantiated claims that may encourage parents to use these devices to monitor infants for medical conditions such as sudden infant death syndrome and potentially deliver unecessary or even harmful interventions [123]. 
Pulmonary health \& environmental exposures

Pulmonary disease wearables take advantage of the fact that most airway abnormalities can be characterized by the abnormal sounds (adventitious sounds). Health Care Originals, Inc. (NY, USA) originally designed a multisensor asthma detection system and later simplified to a single audio-recording system for respiratory assessment in an effort to extend battery life for more practical use [26]. Studies demonstrate that the wearable chest device, ADAMM, can be used to track adolescent asthma [26-28]. In a recent validation study, 84 teens between the ages of 13 and 17 were monitored for 1 week during which the ADAMM device collected audio data to assess the number of cough events, ultimately demonstrating an asthma detection accuracy of $71 \%$ [26]. This study established the device's utility for detection and a modest potential to predict near-future disease progression. Follow-up studies have distinguished asthma, chronic obstructive pulmonary disease, pneumonia, congestive heart failure and lung fibrosis using ADAMM. Wheezing, characterized by narrowing airway and limited airflow, is linked to both asthma and chronic obstructive pulmonary disease while fine crackling, caused by 'explosive opening of small airways,' is linked with pneumonia, congestive heart failure and lung fibrosis [124]. Based on collective results of 16 studies, wheeze and crackle event classification had accuracies between 75.78-100\% and 89-98.15\%, respectively [124]. Airway obstruction caused by anaphylaxis may also soon be detectable through wearable sensors. Project Abbie is a collaborative effort between Harvard's Wyss Institute and the KeepSmilin4Abbie Foundation to develop a wearable device that would be able to detect early reaction signs and automatically administer an injection of epinephrine in response [125].

The large number of potentially important environmental exposures makes developing a single 'exposome' device challenging [126]. Toward the inclusion of multiple sensors to improve the accuracy of pulmonary disease efforts, low-power solutions for integrating personal ozone exposure and volatile organic compound measures are beginning to appear [127]. Other environmental exposure detection devices include patches to measure ultraviolet light and radiation exposure [128].

\section{Regulatory oversight \& economic impact}

FDA oversight on digital health includes categories such as mobile health (mHealth), health information technology, wearable devices, telehealth and telemedicine, and personalized medicine. In September 2017, the FDA announced a digital health software precertification pilot program to focus on software design, validation and maintenance by digital health technology companies. The nine companies selected to participate in this program include Apple (CA, USA), Fitbit, Johnson \& Johnson (CA, USA), Pear Therapeutics (CA, USA), Phosphorous (NY, USA), Roche (CA, USA), Samsung (SKR), Tidepool (CA, USA) and Verily. Wireless medical devices are overseen jointly by the Federal Communications Commission and the FDA to regulate the convergence of medical devices with connectivity and consumer technology and by the Centers for Medicare and Medicaid Services (CMS) to determine costs and market regulation [129]. An abundance of standards are in place and accessible via the FDA Recognized Consensus Standards database [129,130]. Most devices described in this review are regulated as Wireless Medical Telemetry Systems. The FDA describes Wireless medical telemetry as a system generally used to monitor a patient's vital signs (e.g., pulse and respiration) using radio frequency communication, which has the advantage of allowing patient movement without restricting patients to a bedside monitor with a hard-wired connection.

Commercially available motion sensing, physiological and biochemical wearable sensors have been adopted for use in both consumer and clinical healthcare. Although consumer-grade research devices have been primarily adopted for fitness, medical professionals are beginning to find utility in information collected by these devices. Currently, there is a wide effort to integrate information from these devices into electronic health records. Several devices that have been recently approved by the FDA or that are currently undergoing FDA approval have the potential to dramatically change healthcare landscape given their potential for use in and out of the clinic for health and disease management.

With the global wearable devices market anticipated to reach $\$ 19.5$ billion in 2021 [131], wearable technology has the momentum to dramatically alter our current healthcare economics landscape. Replacing just one in five outpatient consultations and home health visits with digital visits would alone save $\$ 40$ billion a year in healthcare costs [132]. In a study involving patients with implantable cardiovascular devices $(n=43)$, remote monitoring decreased healthcare costs for those patients by $25 \%$ [133]. If wearables were to replace even a few of the many costly, gold standard tests and services mentioned in this review, it becomes clear that this kind of combined savings could effectively reduce much of the healthcare burden. 


\section{Conclusion}

Wearable sensors have an extremely broad variety of applications to specific areas of medicine, enabling monitoring of acute and chronic conditions and overall health both in and outside of the clinical setting. Ongoing studies are exploring ways in which this longitudinal data can be used to change the paradigm of medicine from a reactive to a proactive health system. The Precision Medicine Initiative, the Google Baseline Project, MyHeartCounts, the Stanford Azumio Activity Inequality study and the Health ePeople study are four large-scale initiatives to collect longitudinal wearable device data alongside other types of biomedical data, including clinical and biomolecular information about an individual [16,134-136]. The hope is that these studies will uncover new ways in which we can use wearable device data to improve healthcare. As described above, there are already several findings to support the use of wearable sensors combined with tailored algorithms for continuous, longitudinal monitoring, for example, for detection of abnormal heart conditions, hypertension, diabetes, for example (Figure 1C). Further, these tools can be used in specific settings to target extreme healthcare costs, for example, by reducing emergency room visits and hospital readmission rates, improving postsurgical and rehabilitation outcomes, assisting with aging in-home, and preventing serious, preventable and costly medical events (deemed 'never events' by CMS) (Figure 2A) [137]. Wearables can inform ongoing or new symptoms, such as medication side effects or interactions, and may be able to provide automated just-in-time interventions. Additional benefits of wearable sensors in the field setting include their applicability in environments where rapid medical care is needed and often not readily available, for example, at the scene of accidents, in military settings, athletics and in resource-constrained environments (Figure 2) [36,138-140]. Wearable and environmental sensors promise to improve information collection and transfer rapidly, potentially to off-site experts and specially designed algorithms enable automated and real-time medical decision support in time- and resource-constrained environments (Figure 2B).

\section{Challenges \& future directions}

There are several limitations to the use of wearable technology in healthcare (Figure 2D). High device cost is a concern that may hinder the accessibility of this consumer healthcare technology to benefit only those who can afford the devices. Further, many devices are not compatible across platforms (e.g., iOS and Android smart phone operating systems) and there is a lack of data standards which limits their broad use and ubiquity. The time needed to choose and properly set up a device, and remembering to charge the device are also potential barriers to proper use. The acceptability of these devices and potential stigma associated with them varies by community and may also be a factor in dividing who benefits from these technologies [141].

Most importantly, the accuracy of the devices and evidence that they do in fact improve health outcomes must be established with large clinical and field trials. It is common to find unsubstantiated scientific claims with no reference to peer-reviewed studies on commercial wearable device company websites and advertising materials that can set a dangerous precedent if inaccurate or misinterpreted data is factored into medical decision making. False negatives can cause a potentially fatal condition to be missed while false positives can lead to overtreatment and/or anxiety. Inaccuracy of activity trackers may also lead individuals to overestimate their level of physical activity, limiting their effectiveness for lifestyle interventions [142,143]. Shcherbina et al. demonstrated that most wrist-worn devices adequately measure HR in laboratory-based activities, but poorly estimate energy expenditure and caution the use of those measurements as part of health improvement programs [144]. Leth et al. demonstrated that step counts are most accurate at slow walking speeds [145]. While useful for just-in-time interventions, real-time delivery of results should be carefully considered to ensure that accurate data is presented at the right way and at the appropriate time to avoid potentially harmful interventions and patient confusion and anxiety. Training should be provided to health practitioners to increase awareness of the utilities and limitations of wearable technologies [146]. New tools should be developed to assist with the interpretation of wearable device data in a clinical setting. Finally, it is important to further evaluate the emotional burden and increased distraction and technology addiction that wearables can inflict, potentially causing more harm than good.

New and extremely novel wearable sensors are being developed that build upon movement, physiological and biochemical sensors in existence to improve disease detection and prognostication through changes in physiology over time or as a result of a specific treatment or intervention. Mechanical properties of soft tissues were recently monitored via wearables to determine spatiotemporal changes in viscoelasticity of basal cell carcinoma lesions [147]. Wearable oxygen diffusion sensors have been used to track wound healing, and moisture sensors can inform better timing of wound dressing changes $[148,149]$. Within $5-10$ years, we expect that biochemical sensing technologies 
for continuous biofluid analysis (sweat, tears, breath vapor, etc.) will dramatically improve based on a number of early-stage wireless and sufficiently miniaturized devices that demonstrate high potential as wearable sensors.

Kulkarni et al. developed a rapid (down to $0.1 \mathrm{~s}$ ) and sensitive (down to 1 part per billion) nanoelectric graphene vapor sensor that can continuously monitor a wide range of vapor analytes (e.g., nitric oxide, oxygen) from breath or the environment to monitor for illness or harmful environmental exposures [150,151]. The form factor is expected to be a badge that can be worn on clothing. BreathLink is another breath analysis technology to identify women with breast cancer and pulmonary tuberculosis, but this technology is not yet in a wearable form factor $[152,153]$. Wristbands to detect environmental exposures have been successful but require off-site analysis using gas chromatography on returned wristbands [154]. The need for off-site analysis and replacement devices or device cartridges has posed a limitation for longitudinal studies using exposome devices. There are some exposome devices, however, that are beginning to address that [155]. The BACtrack Skyn (CA, USA) comes in the form of either a BACtrack smart watch or a wristband for an Apple watch; both offer continuous blood-alcohol content monitoring through transdermal sensors that measure ethanol vapor at the skin's surface [155].

Several research groups have recently developed mechanically flexible and fully integrated wearable sweat sensors (in wristband, temporary tattoo, eyeglasses, mouthguard) for analysis of biofluid metabolites and electrolytes (e.g., glucose, lactate, sodium, potassium) and $\mathrm{pH}$ sensing [156-160]. The wristband device also measures skin temperature for calibration, and that data is also useful in analyzing health as described earlier $[126,156]$. This device was recently tested for cystic fibrosis diagnosis and preliminary investigation of the blood/sweat glucose correlation [161].

Integrating data collection, analytics and intervention onto one device platform is one of the ultimate goals to develop a fully integrated and independently operating medical device system (Figure 2E). This type of device has been explored most recently through closed-loop insulin delivery systems for Type 1 diabetics, and broader applications are forthcoming [162]. Wearable systems enabling stimulus-induced drug delivery (with the stimulus being mechanical changes or signals based on biosensor data analysis) that enable a dose- and spatiotemporally controlled sustained release of therapeutics have been recently explored [163]. Microneedle arrays for transcutaneous administration of small drugs, hormones or vaccines to the body are just a few examples of how this technology may be employed for just-in-time interventions [164].

Although immense progress has been made over the past several decades in wearable sensing technologies and even more recently toward their applications in healthcare, significant limitations must be addressed for wearable biosensing to achieve its full potential of seamless integration into healthcare.

Improvements in sensor accuracy through hardware and software will enable more accurate detection of health changes over time. Improved battery life and wireless communication (e.g., direct communication to wireless networks) will enable longer monitoring periods, increased utility in resource-limited settings, higher sampling rates (when demonstrated to be necessary for specific applications) and increased participation in the broader population because device charging will become a less frequent need (Figure 2B).

On the regulatory side, more strict oversight has been called for to prevent potentially harmful consequences that can arise from device misuse, inaccurate data or misinterpretation of data from these devices (Figure 2D) [121]. The lack of publicly available evidence supporting the safety, accuracy, effectiveness or role of these monitors hinders their use in clinical and healthcare settings. This can be addressed by vetting of wearable technologies through randomized controlled trials with results available in peer-reviewed publications as well as FDA approval prior to use in consumer health or the clinic. Unsubstantiated claims that overstate the testing accuracy and indications for the device are commonly made by wearable device companies to improve sales, and further regulations are needed to ensure their appropriate use and to evaluate the potential harm that may result from medical interventions or overdiagnosis based on the use of these devices. In Table 1, we demonstrate a variety of wearable devices and cite references to peer-reviewed studies, where available. FDA-regulated devices like CGMs have substantially more clinical trials establishing the safety and efficacy of these devices in healthcare (only the most recent/impactful studies are referenced for brevity), but consumer-grade FDA devices have limited evidence of their utility in healthcare.

The tripartite regulation of wearable devices by the federal government through the FDA, Federal Communications Commission and CMS creates challenges for device manufacturers and vendors whereby regulations can be difficult to adhere to due to the different, and sometimes divergent, objectives of these agencies. Importantly, it will be necessary for CMS to define the reimbursement structure for these devices once CMS determines that the device provides 'reasonable and necessary' care. 
On the hardware side, improvements in technology are needed to enhance the usability of devices. There are often conflicting technological constraints in device design. Pantelopoulos and Bourbakis created a scoring system from the perspective of the patient/wearer, the manufacturer and supervising physician for wearables found that many systems score low on wearability, because sensor, battery and on-body hardware size tends to be too bulky in weight and size [165]. Improved battery life/energy scavenging technologies/energy utilization and multiplexing of sensing modalities and the development and scalable manufacturing of flexible form factors (e.g., stretchable sensing materials, smart textiles) will continue to address this challenge [166]. Further, ensuring that the sensors are tuned to capture physiologically relevant ranges of data and are placed on the appropriate body location for capturing that information will improve utility of the data and will also reduce power consumption from the collection of irrelevant data [167]. In considering human factors, the method of collecting and accessing information from the device should be continuous and passive so that there is no obtrusive user interaction required, which limits user engagement and compliance with the device and user retention, and so that data is collected in the appropriate setting for detecting physiological phenomena of interest.

On the software and data use side, algorithms must be improved to ensure that actionable insights can be derived from the device data. Challenges to addressing this include the wide range of device and system architectures and the proprietary nature of many analytic platforms which limit their generalizability. By creating data collection and transfer standards and developing more generalizable analytic platforms that can send useful information for the patient and physician directly to the electronic health record, it will become easier to use wearable sensor data in a meaningful way in the clinic and to develop closed loop intervention systems [168]. The further integration of wearable devices into the Internet of Things will improve the clinical interpretation and actionability of the device data in the broader context of an individual's lifestyle (Figure 2B \& E). Proper encryption and authentication mechanisms are needed to ensure that privacy is maintained for protected health and personal information.

Addressing all of the above challenges will improve utility and expand the market of these devices and will ultimately will improve long-term health monitoring outside of the clinic as well as healthcare access in lowresource settings and marginalized communities.

Executive summary

Typical wearable devices with applications in health

- A broad range of consumer, clinical and research-grade wearable sensors exist today that are already revolutionizing the healthcare landscape.

Wearable devices used for general health management

- Wearable sensors are currently being used to improve care both inside and outside of the hospital.

Wearable biosensors revolutionizing specific fields of healthcare outside of the hospital \& clinic

- Ambulatory, remote monitoring using wearable sensors is being used for longitudinally tracking conditions related to metabolic, cardiovascular and gastrointestinal disorders; sleep, neurology, movement disorders and mental health; maternal, pre- and neo-natal care; and pulmonary health and environmental exposures.

Challenges \& future directions

- Many technical and regulatory hurdles remain, that when addressed, will improve the adoption of wearable sensors for healthcare.

Acknowledgements

Authors would like to thank JY Galfi and A Rajagopal for their insightful feedback on this manuscript.

Financial \& competing interests disclosure

J Dunn and R Runge are funded by the Mobilize Center, a NIH Big Data to Knowledge Center of Excellence (NIH U54 EB020405). M

Snyder is a cofounder of Personalis, Qbio, Sensomics and January. He is on the scientific advisory of these companies and Genapsys.

The authors have no other relevant affiliations or financial involvement with any organization or entity with a financial interest in or financial conflict with the subject matter or materials discussed in the manuscript apart from those disclosed.

No writing assistance was utilized in the production of this manuscript.

Open access

This work is licensed under the Attribution-NonCommercial-NoDerivatives 4.0 Unported License. To view a copy of this license, visit http://creativecommons.org/licenses/by-nc-nd/4.0/ 


\section{References}

Papers of special note have been highlighted as: $\bullet$ of interest; $\bullet \bullet$ of considerable interest

1. Tamsin M. Wearable biosensor technologies. Int. J. Innov. Sci. Res. 13, 697-703 (2015).

2. Boscari F, Galasso S, Acciaroli G et al. Head-to-head comparison of the accuracy of Abbott FreeStyle Libre and Dexcom G5 mobile. Nutr. Metab. Cardiovasc. Dis. 28(4), 425-427 (2018).

3. Thompson H, Lunt H, Fleckney C, Soule S. Insulin degludec overdose in an adolescent with Type 1 diabetes: proactive management including monitoring using the Freestyle Libre flash glucose monitoring system. Endocrinol. Diabetes Metab. Case Rep. doi: 10.1530/EDM-18-0044 (2018) (Epub ahead of print).

4. Massa GG, Gys I, Op 't Eyndt A et al. Evaluation of the FreeStyle ${ }^{\circledR}$ Libre Flash Glucose Monitoring System in children and adolescents with Type 1 diabetes. Horm. Res. Paediatr. 89(3), 189-199 (2018).

5. Matuleviciene V, Joseph JI, Andelin M et al. A clinical trial of the accuracy and treatment experience of the Dexcom G4 sensor (Dexcom G4 system) and Enlite sensor (guardian REAL-time system) tested simultaneously in ambulatory patients with Type 1 diabetes. Diabetes Technol. Ther. 16(11), 759-767 (2014).

6. Park J, Kim J, Kim SY et al. Soft, smart contact lenses with integrations of wireless circuits, glucose sensors, and displays. Sci. Adv. 4(1), eaap9841 (2018).

7. Kim J, Kim M, Lee MS et al. Wearable smart sensor systems integrated on soft contact lenses for wireless ocular diagnostics. Nat. Commun. 8, 14997 (2017).

8. Schwartz FL, Marling CR, Bunescu RC. The promise and perils of wearable physiological sensors for diabetes management. J. Diabetes Sci. Technol. doi:10.1177/1932296818763228 1932296818763228 (2018) (Epub ahead of print).

9. 3,215,136 HOLTER RES FOUNDATION: Inc. US3215136A, (1965).

10. Teplitzky BA, McRoberts M. Fully-automated ventricular ectopic beat classification for use with mobile cardiac telemetry. Presented at: IEEE 15th International Conference Wearable and Implantable Body Sensor Networks (BSN) NV, USA, 4-7 March 2018.

11. Nandakumar S. Long-term remote monitoring of vital signs using a wireless patch sensor. Presented at: IEEE Health Innovations and Point-of-Care Technologies Conference Seattle, WA, USA (8-10 October 2014).

12. Vandenberk T, Stans J, Mortelmans C et al. Clinical validation of heart rate apps: mixed-methods evaluation study. JMIR Mhealth Uhealth 5(8), e129 (2017).

13. Orchard J, Lowres N, Freedman SB et al. Screening for atrial fibrillation during influenza vaccinations by primary care nurses using a smartphone electrocardiograph (iECG): a feasibility study. Eur. J. Prev. Cardiol. 23(Supp. 2), 13-20 (2016).

14. Lau JK, Lowres N, Neubeck L et al. iPhone ECG application for community screening to detect silent atrial fibrillation: a novel technology to prevent stroke. Int. J. Cardiol. 165(1), 193-194 (2013).

15. Halcox JPJ, Wareham K, Cardew A et al. Assessment of remote heart rhythm sampling using the AliveCor heart monitor to screen for atrial fibrillation: the REHEARSE-AF study. Circulation 136(19), 1784-1794 (2017).

16. Tison GH, Sanchez JM, Ballinger B et al. Passive detection of atrial fibrillation using a commercially available smartwatch. JAMA Cardiol. doi:10.1001/jamacardio.2018.0136 (2018) (Epub ahead of print).

•• The first instance of automated detection of cardiac events using wearable sensors.

17. Shilaih M, Clerck V, Falco L, Kubler F, Leeners B. Pulse rate measurement during sleep using wearable sensors, and its correlation with the menstrual cycle phases, a prospective observational study. Sci. Rep. 7(1), 1294 (2017).

- Demonstrates that traditional methods of clinical measurements are insufficient to capture temporal and cyclical biological variation.

18. Altini M, Rossetti E, Rooijakkers $\mathrm{M}$ et al. Combining electrohysterography and heart rate data to detect labour. Presented at: Biomedical \& Health Informatics (BHI), 2017 IEEE EMBS International Conference Orlando-, FL, USA (16-19 February 2017).

19. Heinemann L, Freckmann G, Ehrmann D et al. Real-time continuous glucose monitoring in adults with Type 1 diabetes and impaired hypoglycaemia awareness or severe hypoglycaemia treated with multiple daily insulin injections (HypoDE): a multicentre, randomised controlled trial. Lancet 391(10128), 1367-1377 (2018).

20. Poh MZ, Loddenkemper T, Reinsberger $\mathrm{C}$ et al. Convulsive seizure detection using a wrist-worn electrodermal activity and accelerometry biosensor. Epilepsia 53(5), e93-e97 (2012).

21. Dolgin E. Dressed to detect. Nature 511(Suppl. 7508), S16-S17 (2014).

22. Kroll RR, Mckenzie ED, Boyd JG et al. Use of wearable devices for post-discharge monitoring of ICU patients: a feasibility study. J. Intensive Care 5, 64 (2017).

23. Spiegel BM, Kaneshiro M, Russell MM et al. Validation of an acoustic gastrointestinal surveillance biosensor for postoperative ileus. J. Gastrointest. Surg. 18(10), 1795-1803 (2014).

24. G-Tech Medical. Current Studies (2018). www.gtechmedical.com/clinical-studies.php

25. Dua MM, Malhotra L, Navalgund AR, Axelrod S, Triadafilopoulos G, Visser B. Monitoring of gastric myoelectric activity after pancreaticoduodenectomy. Gastroenterology 152(5), S1293-S1294 (2017). 
26. Rhee H, Belyea MJ, Sterling M, Bocko MF. Evaluating the validity of an automated device for asthma monitoring for adolescents: correlational design. J. Med. Internet Res. 17(10), e234 (2015).

27. Rhee H, Miner S, Sterling M, Halterman JS, Fairbanks E. The development of an automated device for asthma monitoring for adolescents: methodologic approach and user acceptability. JMIR Mhealth Uhealth 2(2), e27 (2014).

28. Sterling M, Rhee H, Bocko M. Automated cough assessment on a mobile platform. J. Med. Eng. (2014). www.hindawi.com/journals/jme/2014/951621/cta/

29. Turakhia MP, Hoang DD, Zimetbaum P et al. Diagnostic utility of a novel leadless arrhythmia monitoring device. Am. J. Cardiol. 112(4), 520-524 (2013).

30. Solomon MD, Yang J, Sung SH et al. Incidence and timing of potentially high-risk arrhythmias detected through long term continuous ambulatory electrocardiographic monitoring. BMC Cardiovasc. Disord. 16, 35 (2016).

31. Sinha M, Mckeon KM, Parker $S$ et al. A comparison of time delay in three continuous glucose monitors for adolescents and adults. J. Diabetes Sci. Technol. 11(6), 1132-1137 (2017).

32. Topouchian J, Agnoletti D, Blacher J et al. Validation of four devices: Omron M6 Comfort, Omron HEM-7420, Withings BP-800, and Polygreen KP-7670 for home blood pressure measurement according to the European Society of Hypertension International Protocol. Vasc. Health Risk Manag. 10, 33 (2014).

33. Vienneau J, Bauman J, Nigg S, Nigg BM, Jarvis SE. Investigating the effects of the SurroGait Rx ${ }^{\top M}$ device on postural stability, gait, and MSIS-29 outcomes in people with multiple sclerosis. Biomed. Open Access J. Med. Clin. Res. 2(1) (2018).

34. Schlachetzki JC, Barth J, Marxreiter F et al. Wearable sensors objectively measure gait parameters in Parkinson's disease. PLoS ONE 12(10), e0183989 (2017).

35. De Zambotti M, Rosas L, Colrain IM, Baker FC. The sleep of the ring: comparison of the OURA sleep tracker against polysomnography. Behav. Sleep Med. doi:10.1080/15402002.2017.1300587 (2017) (Epub ahead of print).

36. O'Connor KL, Rowson S, Duma SM, Broglio SP. Head-impact-measurement devices: A systematic review. J. Athl. Train. 52(3), 206-227 (2017).

37. Martinez HP, Bengio Y, Yannakakis GN. Learning deep physiological models of affect. IEEE Comput. Intel. Mag. 8(2), 20-33 (2013).

38. Chung MK, Szymkiewicz SJ, Shao M et al. Aggregate national experience with the wearable cardioverter-defibrillator: event rates, compliance, and survival. J. Am. College Cardiol. 56(3), 194-203 (2010).

39. Casson AJ, Galvez AV, Jarchi D. Gyroscope vs. accelerometer measurements of motion from wrist PPG during physical exercise. ICT Express 2(4), 175-179 (2016).

40. Li X, Dunn J, Salins D et al. Digital health: tracking physiomes and activity using wearable biosensors reveals useful health-related information. PLoS Biol. 15(1), e2001402 (2017).

-. The first deep phenotyping study that used wearable sensing to detect health events.

41. Ballinger B, Hsieh J, Singh A et al. DeepHeart: semi-supervised sequence learning for cardiovascular risk prediction. arXiv (2018). https://arxiv.org/abs/1802.02511

42. Lim WK, Davila S, Teo JX et al. Beyond fitness tracking: The use of consumer-grade wearable data from normal volunteers in cardiovascular and lipidomics research. PLoS Biol. 16(2), e2004285 (2018).

43. Price ND, Magis AT, Earls JC et al. A wellness study of 108 individuals using personal, dense, dynamic data clouds. Nat. Biotechnol. 35(8), 747-756 (2017).

44. Chen R, Mias GI, Li-Pook-Than J et al. Personal omics profiling reveals dynamic molecular and medical phenotypes. Cell 148(6), 1293-1307 (2012).

45. Piening BD, Zhou W, Contrepois K et al. Integrative personal omics profiles during periods of weight gain and loss. Cell Syst. 6(2), $157-170$ e158 (2018)

46. Rego S, Dagan-Rosenfeld O, Zhou W et al. High frequency actionable pathogenic exome mutations in an average-risk cohort. bioRxiv doi: https://doi.org/10.1101/151225 (2017) (E pub ahead of print).

47. Steinhubl SR, Feye D, Levine AC, Conkright C, Wegerich SW, Conkright G. Validation of a portable, deployable system for continuous vital sign monitoring using a multiparametric wearable sensor and personalised analytics in an Ebola treatment centre. BMJ Global Health 1(1), e000070 (2016).

48. Selvaraj N, Narasimhan R. Detection of sleep apnea on a per-second basis using respiratory signals. Presented at: Engineering in Medicine and Biology Society (EMBC), 2013 35th Annual International Conference of the IEEE. Osaka, Japan, (3-7 July 2013).

49. Selvaraj N, Narasimhan R. Automated prediction of the apnea-hypopnea index using a wireless patch sensor. Presented at: Engineering in Medicine and Biology Society (EMBC), 2014 36th Annual International Conference of the IEEE. IL, USA (26-30 August 2014).

50. Narasimhan R. Skin-contact sensor for automatic fall detection. Presented at: Engineering in Medicine and Biology Society (EMBC), 2012 Annual International Conference of the IEEE. CA, USA (28 August-1 September 2012). 
51. Chan AM, Selvaraj N, Ferdosi N, Narasimhan R. Wireless patch sensor for remote monitoring of heart rate, respiration, activity, and falls. Presented at: Engineering in Medicine and Biology Society (EMBC), 2013 35th Annual International Conference of the IEEE. Osaka, Japan, (3-7 July 2013).

52. Claudio D, Velázquez MA, Bravo-Llerena W, Okudan GE, Freivalds A. Perceived usefulness and ease of use of wearable sensor-based systems in emergency departments. IIE Trans. Occup. Ergon. Hum. Factors 3(3-4), 177-187 (2015).

53. Levin S, Toerper M, Hamrock E et al. Machine-learning-based electronic triage more accurately differentiates patients with respect to clinical outcomes compared with the emergency severity index. Ann. Emerg. Med. doi:10.1016/j.annemergmed.2017.08.005 (2017) (Epub ahead of print).

54. Moreno S, Quintero A, Ochoa C, Bonfante M, Villareal R, Pestana J. Remote monitoring system of vital signs for triage and detection of anomalous patient states in the emergency room. Presented at: 2016 XXI Symposium on Signal Processing, Images and Artificial Vision (STSIVA). Bucaramanga, Colombia, (30 August-2 September 2016).

55. Devita MA, Hillman K, Bellomo R et al. Presented at: Textbook of Rapid Response Systems: Concept and Implementation. Springer, Switzerland, (2017). https://link.springer.com/book/10.1007\%2F978-3-319-39391-9

56. Aboukhalil A, Nielsen L, Saeed M, Mark RG, Clifford GD. Reducing false alarm rates for critical arrhythmias using the arterial blood pressure waveform. J. Biomed. Inform. 41(3), 442-451 (2008).

57. Lewy H. Wearable technologies - future challenges for implementation in healthcare services. Healthc. Technol. Lett. 2(1), 2-5 (2015).

58. Fotiadis DI, Glaros C, Likas A. Wearable medical devices. Wiley Encyclopedia of Biomedical Engineering Wiley, NJ, USA (2006). Wiley https://doi.org/10.1002/9780471740360.ebs1326

59. Kohn MS, Sun J, Knoop S et al. IBM's health analytics and clinical decision support. Yearb. Med. Inform. 9, 154-162 (2014).

60. Wright A, Sittig DF, Ash JS, Sharma S, Pang JE, Middleton B. Clinical decision support capabilities of commercially-available clinical information systems. J. Am. Med. Inform. Assoc. 16(5), 637-644 (2009).

61. Yin H, Jha NK. A health decision support system for disease diagnosis based on wearable medical sensors and machine learning ensembles. IEEE Transact. Multiscale Comput. Syst. 3(4), 228-241 (2017).

62. Sittig DF, Wright A, Osheroff JA et al. Grand challenges in clinical decision support. J. Biomed. Inform. 41(2), 387-392 (2008).

63. Barrett PM, Komatireddy R, Haaser S et al. Comparison of 24-hour Holter monitoring with 14-day novel adhesive patch electrocardiographic monitoring. Am. J. Med. 127(1), 95, e11-e97 (2014).

64. Semi Supervised Sequence Learning for Continuous Digital Biomarkers. Neural information processing systems, machine learning for healthcare workshop. (2016). www.nipsml4hc.ws/posters

65. Cardiovascular risk stratification using off-the-shelf wearables and a multi-task deep learning algorithm. American Heart Association Scientific Sessions. (2017). https://doi.org/10.1161/circ.136.suppl_1.21042

66. Lee H, Chung H, Ko H et al. Dedicated cardiac rehabilitation wearable sensor and its clinical potential. PLoS ONE 12(10), e0187108 (2017).

67. Nwankwo T, Yoon SS, Burt V, Gu Q. Hypertension among adults in the United States: National Health and Nutrition Examination Survey, 2011-2012. NCHS Data Brief(133), 1-8 (2013).

68. Wexler R. Ambulatory blood pressure monitoring in primary care. South Med. J. 103(5), 447-452 (2010).

69. Hermida RC, Ayala DE, Mojon A, Fernandez JR. Decreasing sleep-time blood pressure determined by ambulatory monitoring reduces cardiovascular risk. J. Am. Coll. Cardiol. 58(11), 1165-1173 (2011).

70. Burkard T, Mayr M, Winterhalder C, Leonardi L, Eckstein J, Vischer AS. Reliability of single office blood pressure measurements. Heart doi:10.1136/heartjnl-2017-312523 (2018) (Epub ahead of print).

71. Asayama K, Thijs L, Brguljan-Hitij J et al. Risk stratification by self-measured home blood pressure across categories of conventional blood pressure: a participant-level meta-analysis. PLoS Med 11(1), e1001591 (2014).

72. Pickering TG. The clinical significance of diurnal blood pressure variations. Dippers and nondippers. Circulation 81(2), 700-702 (1990).

73. Mahabala C, Kamath P, Bhaskaran U, Pai ND, Pai AU. Antihypertensive therapy: nocturnal dippers and nondippers. Do we treat them differently? Vasc. Health Risk Manag. 9, 125-133 (2013).

74. National Heart Foundation and High Blood Pressure Research Council of Australia Ambulatory Blood Pressure Monitoring Consensus Committee. Ambulatory blood pressure monitoring. Aust. Fam. Physician 40(11), 877-880 (2011).

75. Solà J, Proença M, Braun F et al. Continuous non-invasive monitoring of blood pressure in the operating room: a cuffless optical technology at the fingertip. Curr. Dir. Biomed. Eng. 2(1), 267-271 (2016).

76. Mukkamala R, Hahn J-O, Inan OT et al. Toward ubiquitous blood pressure monitoring via pulse transit time: theory and practice. IEEE Transact. Biomed. Eng. 62(8), 1879-1901 (2015).

77. Feakins RM. Obesity and metabolic syndrome: pathological effects on the gastrointestinal tract. Histopathology 68(5), 630-640 (2016).

78. Watanabe S, Hojo M, Nagahara A. Metabolic syndrome and gastrointestinal diseases. J. Gastroenterol. 42(4), 267-274 (2007). 
79. Peery AF, Dellon ES, Lund J et al. Burden of gastrointestinal disease in the United States: 2012 update. Gastroenterology 143(5), 1179-1187 e1171-e1173 (2012).

80. Kaneshiro M, Kaiser W, Pourmorady J et al. Postoperative gastrointestinal telemetry with an acoustic biosensor predicts ileus vs. uneventful GI recovery. J. Gastrointest. Surg. 20(1), 132-139 (2016).

81. Gharibans AA, Kim S, Kunkel D, Coleman TP. High-resolution electrogastrogram: a novel, noninvasive method for determining gastric slow-wave direction and speed. IEEE Transact. Biomed. Eng. doi:10.1109/TBME.2016.2579310 (2016) (Epub ahead of print).

82. Gharibans AA, Smarr BL, Kunkel DC, Kriegsfeld LJ, Mousa HM, Coleman TP. Artifact rejection methodology enables continuous, noninvasive measurement of gastric myoelectric activity in ambulatory subjects. Sci. Rep. 8(1), 5019 (2018).

83. Dagdeviren C, Javid F, Joe P et al. Flexible piezoelectric devices for gastrointestinal motility sensing. Nat. Biomed. Eng. 1(10), 807 (2017).

84. Kaplan KA, Hirshman J, Hernandez B et al. When a gold standard isn't so golden: lack of prediction of subjective sleep quality from sleep polysomnography. Biol. Psychol. 123, 37-46 (2017).

85. Centers for Disease Control and Prevention. Sleep and sleep disorders (2017). www.cdc.gov/sleep/about_us.html

86. Punjabi NM. The epidemiology of adult obstructive sleep apnea. Proc. Am. Thorac. Soc. 5(2), 136-143 (2008).

87. Wu H-T, Wu J-C, Huang P-C et al. Phenotype-based and self-learning inter-individual sleep Apnea screening with a level IV monitoring system. arXiv (2018). https://arxiv.org/abs/1802.09706

88. Horvath K, Aschermann Z, Acs P et al. Is the MDS-UPDRS a good screening tool for detecting sleep problems and daytime sleepiness in Parkinson's disease? Parkinsons Dis. 2014, 806169 (2014).

89. Walker MP. The role of sleep in cognition and emotion. Ann. NY Acad. Sci. 1156, 168-197 (2009).

90. Bianchi MT. Sleep devices: wearables and nearables, informational and interventional, consumer and clinical. Metabolism 84 99-108 (2017).

-91. Kartsch VJ, Benatti S, Schiavone PD, Rossi D, Benini L. A sensor fusion approach for drowsiness detection in wearable ultra-low-power systems. Inform. Fusion 43, 66-76 (2018).

92. Murray CJ, Atkinson C, Bhalla K et al. The state of US health, 1990-2010: burden of diseases, injuries, and risk factors. JAMA 310(6), 591-608 (2013).

93. US Department of Veteran Affairs. VA research on depression. (2018). www.research.va.gov/pubs/docs/va_factsheets/Depression.pdf

94. Runyan JD, Steenbergh TA, Bainbridge C, Daugherty DA, Oke L, Fry BN. A smartphone ecological momentary assessment/intervention 'app' for collecting real-time data and promoting self-awareness. PLoS ONE 8(8), e71325 (2013).

95. Shiffman S, Stone AA, Hufford MR. Ecological momentary assessment. Annu. Rev. Clin. Psychol. 4, 1-32 (2008).

96. Kim J, Nakamura T, Kikuchi H, Sasaki T, Yamamoto Y. Co-variation of depressive mood and locomotor dynamics evaluated by ecological momentary assessment in healthy humans. PLoS ONE 8(9), e74979 (2013).

-97. Gentili C, Valenza G, Nardelli M et al. Longitudinal monitoring of heartbeat dynamics predicts mood changes in bipolar patients: a pilot study. J. Affect. Disord. 209, 30-38 (2017).

98. Faedda GL, Ohashi K, Hernandez M et al. Actigraph measures discriminate pediatric bipolar disorder from attention-deficit/hyperactivity disorder and typically developing controls. J. Child Psychol. Psychiatry. 57(6), 706-716 (2016)

99. Torrado JC, Gomez J, Montoro G. Emotional self-regulation of individuals with autism spectrum disorders: smartwatches for monitoring and interaction. Sensors (Basel) 17(6), 1359 (2017).

100. Hashemi J, Tepper M, Vallin Spina T et al. Computer vision tools for low-cost and noninvasive measurement of autism-related behaviors in infants. Autism Res. Treat. 2014, 935686 (2014).

101. Centers for Disease Control and Prevention. Epilepsy fast facts. (2018). www.cdc.gov/epilepsy/about/fast-facts.htm

102. WHO. Improving access to epilepsy care. WHO doi:/entity/mental_health/neurology/epilepsy/en/index.html (2018) (Epub ahead of print).

103. Poh M-Z, Loddenkemper T, Reinsberger $\mathrm{C}$ et al. Autonomic changes with seizures correlate with postictal EEG suppression. Neurology 78(23), 1868-1876 (2012).

104. Rice JB, Desai U, Cummings AKG, Birnbaum HG, Skornicki M, Parsons NB. Burden of diabetic foot ulcers for medicare and private insurers. Diabetes Care 37(3), 651-658 (2014).

105. Najafi B, Ron E, Enriquez A, Marin I, Razjouyan J, Armstrong DG. Smarter sole survival: will neuropathic patients at high risk for ulceration use a smart insole-based foot protection system? J. Diabetes Sci. Technol. 11(4), 702-713 (2017).

106. Gia TN, Sarker VK, Tcarenko I et al. Energy efficient wearable sensor node for IoT-based fall detection systems. Microprocess. Microsyst. 56, 34-46 (2018).

107. Ojetola O, Gaura EI, Brusey J. Fall detection with wearable sensors-safe (smart fall detection). Presented at: Intelligent Environments (IE), 2011 7th International Conference. Nottingham, UK, (25-28 July 2011).

108. Centers for Disease Control and Prevention. Reproductive health: pregnancy mortality surveillance service (2017). www.cdc.gov/reproductivehealth/maternalinfanthealth/pmss.html 
109. Centers for Disease Control and Prevention. Reproductive health: preterm birth (2017). www.cdc.gov/reproductivehealth/maternalinfanthealth/pretermbirth.htm

110. Kuklina EV, Ayala C, Callaghan WM. Hypertensive disorders and severe obstetric morbidity in the United States. Obstet. Gynecol. 113(6), 1299-1306 (2009).

111. Albrecht SS, Kuklina EV, Bansil P et al. Diabetes trends among delivery hospitalizations in the US, 1994-2004. Diabetes Care 33(4), 768-773 (2010).

112. Kuklina E, Callaghan W. Chronic heart disease and severe obstetric morbidity among hospitalizations for pregnancy in the United States: 1995-2006. Obstet. Anesthesia Digest. 31(4), 216 (2011)

113. Chu SY, Bachman DJ, Callaghan WM et al. Association between obesity during pregnancy and increased use of health care. New Engl. J. Med. 358(14), 1444-1453 (2008).

114. Chandra A, Copen CE, Stephen EH. Infertility and impaired fecundity in the United States, 1982-2010: data from the National Survey of Family Growth. Natl Health Stat. Report, August 14(67), 1-18, (2013).

115. Centers for Disease Control and Prevention. National Center for Health Statistics (2013). www.cdc.gov/nchs/data/nhsr/nhsr067.pdf

116. Dietz PM, Vesco KK, Callaghan WM et al. Postpartum screening for diabetes after a gestational diabetes mellitus-affected pregnancy. Obstet. Gynecol. 112(4), 868-874 (2008).

117. Vesco KK, Dietz PM, Bulkley J et al. A system-based intervention to improve postpartum diabetes screening among women with gestational diabetes. Am. J. Obstet. Gynecol. 207(4), 283. e281-283. e286 (2012).

118. Nicklas JM, Zera CA, Seely EW, Abdul-Rahim ZS, Rudloff ND, Levkoff SE. Identifying postpartum intervention approaches to prevent Type 2 diabetes in women with a history of gestational diabetes. BMC Pregnancy Childbirth 11, 23 (2011).

119. Berglund Scherwitzl E, Gemzell Danielsson K, Sellberg JA, Scherwitzl R. Fertility awareness-based mobile application for contraception. Eur. J. Contracept. Reprod. Health Care 21(3), 234-241 (2016).

120. Altini M, Rossetti E, Rooijakkers M et al. 762: remote labour detection using maternal electrohysterography and heart rate data acquired with a wearable sensor. Am. J. Obstet. Gynecol. 216(1), S441-S442 (2017).

121. Bonafide CP, Jamison DT, Foglia EE. The emerging market of smartphone-integrated infant physiologic monitors. JAMA 317(4), 353-354 (2017).

- Covers an important area of consumer health where inaccurate technologies and improper interpretation of wearable device data may lead to over- or mistreatment and potentially devastating consequences.

122. Tanigasalam V, Vishnu Bhat B, Adhisivam B, Balachander B, Kumar H. Hypothermia detection in low birth weight neonates using a novel bracelet device. J. Matern. Fetal Neo-natal. Med. doi:10.1080/14767058.2018.1443072 1-4 (2018) (Epub ahead of print).

123. Moon RY, Task Force on Sudden Infant Death S. SIDS and other sleep-related infant deaths: evidence base for 2016 updated recommendations for a safe infant sleeping environment. Pediatrics 138(5) pii: e20162940 (2016).

124. Pramono RXA, Bowyer S, Rodriguez-Villegas E. Automatic adventitious respiratory sound analysis: a systematic review. PLoS ONE 12(5), e0177926 (2017).

125. Harvard Wyss Institute. Project ABBIE (2016). https://wyss.harvard.edu/technology/project-abbie/

126. Asimina S, Chapizanis D, Karakitsios S et al. Assessing and enhancing the utility of low-cost activity and location sensors for exposure studies. Environ. Monit. Assess 190(3), 155 (2018).

127. Dieffenderfer J, Goodell H, Mills S et al. Low-power wearable systems for continuous monitoring of environment and health for chronic respiratory disease. IEEE J. Biomed. Health Inform. 20(5), 1251-1264 (2016).

128. Barajas-Carmona JG, Francisco-Aldana L, Morales-Narváez E. Wearable nanoplasmonic patch detecting Sun/UV exposure. Anal. Chem. 89(24), 13589-13595 (2017).

129. Center for Devices and Radiological Health. Wireless medical devices. (2018). www.fda.gov/MedicalDevices/default.htm

130. US Food \& Drug Administration. Recognized consensus standards. (2018). www.accessdata.fda.gov/scripts/cdrh/cfdocs/cfStandards/search.cfm

131. Dangi RT. Wearable medical devices: technologies and global markets 2018. (2017) www.reportlinker.com/p05285115/Wearable-Medical-Devices-Technologies-and-Global-Markets.html

132. Atluri V, Cordina J, Mango P, Velamooret S. How tech-enabled consumers are reordering the healthcare landscape. McKinsey \& Company, (2016). www.mckinsey.com/industries/healthcare-systems-and-services/our-insights/how-tech-enabled-consumers-are-reor dering-the-healthcare-landscape

133. Costa PD, Reis AH, Rodrigues PP. Clinical and economic impact of remote monitoring on the follow-up of patients with implantable electronic cardiovascular devices: an observational study. Telemed. J E Health 19(2), 71-80 (2013).

134. Sankar PL, Parker LS. The Precision Medicine Initiative's All of Us research program: an agenda for research on its ethical, legal, and social issues. Genet. Med. 19(7), 743-750 (2017).

•• The precision medicine initiative's All of Us research program is the largest longitudinal study in the history of the USA which will create a cohort of 1 million volunteers who will contribute their health data, including wearables data, and biospecimens to 
a centralized national database to support precision medicine research. Some of the ethical, legal and social issues associated with the initiative.

135. McConnell MV, Shcherbina A, Pavlovic A et al. Feasibility of obtaining measures of lifestyle from a smartphone app: the MyHeart counts cardiovascular health study. JAMA Cardiol. 2(1), 67-76 (2017).

- The first large-scale study demonstrating that a smartphone-based study of cardiovascular health is feasible and allows rapid, large-scale and detailed assessment of physical activity, fitness and sleep.

136. Althoff T, Hicks JL, King AC, Delp SL, Leskovec J. Large-scale physical activity data reveal worldwide activity inequality. Nature 547(7663), 336 (2017).

137. Centers for Medicare \& Medicaid Services. Eliminating serious, preventable, and costly medical errors - never events (2013). www.cms.gov/Newsroom/MediaReleaseDatabase/Fact-sheets/2006-Fact-sheets-items/2006-2005-2018.html

138. Merrell AJ, Christensen WF, Seeley MK, Bowden AE, Fullwood DT. Nano-composite foam sensor system in football helmets. Ann. Biomed. Eng. 45(12), 2742-2749 (2017).

139. King DA, Hume PA, Gissane C, Clark TN. Similar head impact acceleration measured using instrumented ear patches in a junior rugby union team during matches in comparison with other sports. J. Neurosurg. Pediatr. 18(1), 65-72 (2016).

140. Liu B, Medda A, Woods D, Phelps S, Fain W. The integrated blast effects sensor suite: a rapidly developed, complex, system of systems. Mil. Med. 180(Suppl. 3), 195-200 (2015).

141. Farrington C. Wearable technologies and stigma in diabetes: the role of medical aesthetics. Lancet Diabetes Endocrinol. 4(7), 566 (2016).

142. Wallen MP, Gomersall SR, Keating SE, Wisloff U, Coombes JS. Accuracy of heart rate watches: implications for weight management. PLoS ONE 11(5), e0154420 (2016).

143. Dooley EE, Golaszewski NM, Bartholomew JB. Estimating accuracy at exercise intensities: a comparative study of self-monitoring heart rate and physical activity wearable devices. JMIR Mhealth Uhealth 5(3), e34 (2017).

144. Shcherbina A, Mattsson CM, Waggott D et al. Accuracy in wrist-worn, sensor-based measurements of heart rate and energy expenditure in a diverse cohort. J. Pers. Med. 7(2), 3 (2017).

145. Leth S, Hansen J, Nielsen OW, Dinesen B. Evaluation of commercial self-monitoring devices for clinical purposes: results from the future patient trial, Phase I. Sensors (Basel) 17(1), 211 (2017).

146. Piwek L, Ellis DA, Andrews S, Joinson A. The rise of consumer health wearables: promises and barriers. PLoS Med. 13(2), e1001953 (2016).

147. Dagdeviren C, Shi Y, Joe P et al. Conformal piezoelectric systems for clinical and experimental characterization of soft tissue biomechanics. Nat. Mater. 14(7), 728-736 (2015).

148. Niederauer MQ, Michalek JE, Armstrong DG. A prospective, randomized, double-blind multicenter study comparing continuous diffusion of oxygen therapy to sham therapy in the treatment of diabetic foot ulcers. J. Diabetes Sci. Technol. 11(5), 883-891 (2017).

149. Milne SD, Seoudi I, Al Hamad H et al. A wearable wound moisture sensor as an indicator for wound dressing change: an observational study of wound moisture and status. Int. Wound J. 13(6), 1309-1314 (2016).

150. Kulkarni GS, Zang W, Zhong Z. Nanoelectronic heterodyne sensor: a new electronic sensing paradigm. Acc. Chem. Res. 49(11), 2578-2586 (2016).

151. Kulkarni GS, Reddy K, Zhong Z, Fan X. Graphene nanoelectronic heterodyne sensor for rapid and sensitive vapour detection. Nat. Commun. 5, 4376 (2014).

152. Phillips M, Beatty JD, Cataneo RN et al. Rapid point-of-care breath test for biomarkers of breast cancer and abnormal mammograms. PLoS ONE 9(3), e90226 (2014).

153. Phillips M, Basa-Dalay V, Blais J et al. Point-of-care breath test for biomarkers of active pulmonary tuberculosis. Tuberculosis (Edinb.) 92(4), 314-320 (2012)

154. Donald CE, Scott RP, Blaustein KL et al. Silicone wristbands detect individuals' pesticide exposures in West Africa. R. Soc. Open Sci. 3(8), 160433 (2016).

155. National Institute on Alcohol Abuse and Alcoholism. Director's report on institute activities to the 142nd meeting of the National Advisory Council on Alcohol Abuse and Alcoholism (2018).

www.niaaa.nih.gov/about-niaaa/our-work/advisory-council/directors-reports-council/niaaa-directors-report-institute-12

156. Gao W, Emaminejad S, Nyein HYY et al. Fully integrated wearable sensor arrays for multiplexed in situ perspiration analysis. Nature 529(7587), 509-514 (2016).

157. Dang W, Manjakkal L, Navaraj WT, Lorenzelli L, Vinciguerra V, Dahiya R. Stretchable wireless system for sweat pH monitoring. Biosens. Bioelectron. 107, 192-202 (2018)

158. Sempionatto JR, Nakagawa T, Pavinatto A et al. Eyeglasses based wireless electrolyte and metabolite sensor platform. Lab Chip 17(10), 1834-1842 (2017).

159. Jia W, Bandodkar AJ, ValdéS-Ramírez G et al. Electrochemical tattoo biosensors for real-time noninvasive lactate monitoring in human perspiration. Analytical Chemistry 85(14), 6553-6560 (2013). 
160. Kim J, Imani S, De Araujo WR et al. Wearable salivary uric acid mouthguard biosensor with integrated wireless electronics. Biosens. Bioelectron. 74, 1061-1068 (2015).

161. Emaminejad S, Gao W, Wu E et al. Autonomous sweat extraction and analysis applied to cystic fibrosis and glucose monitoring using a fully integrated wearable platform. Proc. Natl Acad. Sci. USA 114(18), 4625-4630 (2017).

-• A seminal demonstration of how fully integrated wearable platforms with multimodal measurement capabilities can dramatically improve monitoring and intervention capabilities.

162. Stone JY, Haviland N, Bailey TS. Review of a commercially available hybrid closed-loop insulin-delivery system in the treatment of Type 1 diabetes. Ther. Deliv. 9(2), 77-87 (2018).

163. Lu Y, Sun W, Gu Z. Stimuli-responsive nanomaterials for therapeutic protein delivery. J. Control. Release 194, 1-19 (2014).

164. Di J, Yao S, Ye Y et al. Stretch-triggered drug delivery from wearable elastomer films containing therapeutic depots. ACS Nano 9(9), 9407-9415 (2015).

165. Pantelopoulos A, Bourbakis N. A survey on wearable biosensor systems for health monitoring. Conf. Proc. IEEE Eng. Med. Biol. Soc. 2008, 4887-4890 (2008).

166. Kim YS, Lu J, Shih B et al. Scalable manufacturing of solderable and stretchable physiologic sensing systems. Adv. Mater. 29(39) (2017) doi.org/10.1002/adma.201701312

167. Kenry Yeo JC, Lim CT. Emerging flexible and wearable physical sensing platforms for healthcare and biomedical applications. Microsyst. Nanoeng. 2, 16043 (2016).

168. Lebak JW, Yao J, Warren S. HL7-compliant healthcare information system for home monitoring. Conf. Proc. IEEE Eng. Med. Biol. Soc. 5, 3338-3341 (2004). 